\title{
Qualidade de vida e nível de atividade física de profissionais de saúde de unidades de terapia intensiva
}

Rev Bras Ativ Fis Saúde p. 711-719

DOI:

http://dx.doi.org/10.12820/rbafs.v.18n6p711

1 Departamento de Fisioterapia do Hospital Dom Hélder Câmara. Recife-PE.

Quality of life and physical activity level of health workers of intensive care unit

\author{
Afonso Celso de Farias Acioli Neto ${ }^{1}$ \\ Rodrigo Cappato de Araújo ${ }^{2}$ \\ Ana Carolina Rodarti Pitangui ${ }^{2}$ \\ Luciana Carvalho de Menezes ${ }^{3}$ \\ Eduardo Eriko Tenório de França ${ }^{4}$ \\ Emilia Chagas Costa ${ }^{5}$ \\ Flávio Maciel Dias de Andrade ${ }^{1,4}$ \\ Marco Aurélio de Valois Correia Junior ${ }^{2}$
}

2 Universidade de Pernambuco. Petrolina-PE

3 Faculdade Maurício de Nassau. Recife-PE.

4 Universidade Católica de Pernambuco (UNICAP). Recife-PE.

5 Universidade Federal de Alagoas (UFAL). Maceió-AL.

\section{Resumo}

O baixo nível de atividade física aliado ao estresse constante na vida dos profissionais que trabalham em Unidade de Terapia Intensiva (UTI) pode interferir diretamente na qualidade de vida $(\mathrm{QV})$ e na saúde destes indivíduos. O objetivo deste estudo foi verificar se os domínios da qualidade de vida de profissionais de saúde que atuam em UTI's diferem em função do nível de atividade física. Trata-se de um estudo transversal realizado em cinco UTI's da região metropolitana do Recife. O nível de atividade física (NAF) foi avaliado pelo International Physical Activity Questionnaire (IPAQ) e a QV foi analisada mediante o questionário Medical Outcomes Study 36 (SF-36), ambos aplicados em forma de entrevista. Foram considerados inativos $62,2 \%$ da população estudada em um total de 246 profissionais de saúde. Os enfermeiros foram considerados inativos $(78 \%)$, seguidos dos médicos $(75,9 \%)$, fisioterapeutas $(58,3 \%)$ e técnicos de enfermagem $(55,1 \%)$. Os indivíduos considerados ativos apresentaram maiores escores nos domínios capacidade funcional $(86,1 \pm 17,4$ vs $79,0 \pm 20,0 ; \mathrm{p}=0,01)$, vitalidade $(71,2 \pm 18,3$ vs $62,9 \pm 25,0 ; \mathrm{p}=0,01)$ e saúde mental $(80,1 \pm 13,3$ vs $74,0 \pm 14,2 ; \mathrm{p}=0,01)$, quando comparados aos inativos. Os escores de QV diferiram entre os profissionais de saúde classificados como ativos e inativos, sendo verificado melhores níveis de QV nos indivíduos fisicamente ativos.

\section{Palavras-chave}

Unidades de terapia intensiva; Atividade motora; Pessoal de saúde; Doenças profissionais; Qualidade de vida.

\begin{abstract}
The low level of physical activity combined with the constant stress in life of professional who working in the intensive care unit (ICU) can directly affect the quality of life (QOL) of these individuals. The aim of this study was to verify if the domains of quality of life of health professionals who work in ICUs differ according to the level of physical activity. It is a cross-sectional study conducted in five ICUs in the metropolitan region of Recife. The physical activity level (PAL) was evaluated by International Physical Activity Questionnaire (IPAQ) and QOL was assessed by the Medical Outcomes Study 36 (SF-36), both applied as an interview. Were considered inactive 62,2\% of the population studied in a total of 246 health professionals. The nurses were considered inactive (78\%), followed by doctors (75,9\%), physiotherapists (58,3\%) and nursing technicians (55.1\%). Individuals considered active had higher scores in physical functioning $(86,1 \pm 17,4$ vs $79,0 \pm 20,0, p=0,01)$, vitality $(71,2 \pm 18,3$ vs $62,9 \pm 25,0, p=0,01)$ and mental health $(80,1 \pm 13,3$ vs $74,0 \pm 14,2, p=0,01)$, when compared to inactive. The QOL scores differed among health professionals classified as active and inactive, being found higher levels of QOL in physically active individuals.
\end{abstract}

\section{Keywords}

Intensive care unit; Motor Activity; Health Personnel; Occupational Diseases; Quality of life. 


\section{INTRODUÇÃO}

A qualidade de vida (QV) foi definida de maneira geral como a percepção subjetiva do sujeito acerca da sua posição na vida, considerando seus objetivos, expectativas, padrões e preocupações ${ }^{1}$. Por meio de diferentes perspectivas, o termo QV tem sido associado à saúde do indivíduo, considerando principalmente o seu bem-estar emocional, físico e social ${ }^{2,3}$. Nesse sentido, a QV tem sido objeto de avaliação em diferentes áreas, inclusive em pesquisas relacionadas à saúde do trabalhador ${ }^{4-6}$.

Em um âmbito geral, o trabalho tem sido considerado parte importante e essencial na vida de um indivíduo, sendo o principal meio pelo qual este interage de forma produtiva com a sociedade. Entretanto, o processo de globalização associado à necessidade de crescimento econômico tem contribuído para profundas alterações nas condições de trabalho, com o aumento da carga horária, o acúmulo de diferentes funções e a cobrança quase constante por maior produtividade. Assim, esses aspectos poderão favorecer a uma maior exposição a fatores desgastantes e potencializadores dos processos saúde-doença e irão contribuir para modificação do estilo de vida, expondo os sujeitos a fatores de risco para saúde ${ }^{7}$.

Dentro desse contexto, a unidade de terapia intensiva (UTI) surge como um ambiente de trabalho que possui uma rotina caracterizada por situações muita vezes incertas, inesperadas, que exigem alta interdependência e tomadas de decisões permeadas por intervenções complexas ${ }^{6,7}$. Os profissionais que atuam nesse ambiente precisam conviver com o sofrimento, a dor e o risco de morte dos pacientes, fato que impõe a esses profissionais um intenso desgaste físico, mental e psicológico, podendo impactar negativamente na $\mathrm{QV}$.

Além disso, o comprometimento da QV dos profissionais de saúde que atuam em UTIs, seja na dimensão física ou emocional, pode influenciar de maneira negativa a dinâmica do funcionamento desse setor, comprometendo a qualidade do serviço prestado levando a um prejuízo institucional e principalmente, aos pacientes atendidos ${ }^{6}$. Nesse sentido, a avaliação e busca pela melhora da QV desses profissionais torna-se importante, tanto do ponto de vista da promoção à saúde e prevenção de doenças, mas também considerando a possibilidade de melhorar a qualidade do serviço prestado nesse setor.

A manutenção de um estilo de vida saudável associado ao incremento da prática de atividades físicas, sejam essas realiadas no lazer, em atividades domésticas, na locomoção e até mesmo no ambiente de trabalho, podem ser consideradas estratégias importantes para a melhora dos indicadores de saúde e $\mathrm{QV}^{8,9}$.

$\mathrm{A} Q \mathrm{QV}$ e a atividade física (AF) estão inter-relacionadas, de modo que a prática regular de atividade física acarreta em ganhos na saúde, melhorando a QV do indivíduo. Pessoas fisicamente ativas apresentam maior vigor e vontade de realizar suas atividades diárias e profissionais, além de demonstrar maior energia e menor cansaço, e um melhor nível cognitivo, colaborando para a melhora das relações interpessoais, tais como familiar e socialização no trabalho ${ }^{8,10}$.

Vários estudos têm apresentado atenção especial para a análise do nível de atividade física (NAF) em diferentes populações ${ }^{6,11,12}$. No entanto, pouco se tem estudado sobre o NAF e a QV nos diferentes profissionais que trabalham em UTI. Dessa forma, considerando o impacto negativo das condições de trabalho na saúde e $\mathrm{QV}$ desses profissionais, as subsequentes repercussões na qualidade do serviço prestado e a possibilidade de uso da AF como estratégia para melhora desse quadro, buscou-se nesse estudo avaliar se os domínios da qualidade de vida de profissionais de saúde que atuam em UTI diferem em função do nível de atividade física. 


\section{MÉTODOS}

Trata-se de um estudo transversal realizado com profissionais de saúde que atuam nas UTI's clínicas de cinco hospitais da região metropolitana do Recife (RMR). Este estudo foi aprovado pelo comitê de ética em pesquisa da fundação Altino Ventura sob o protocolo $\mathrm{N}^{\circ} 032 / 2010$ e todos os entrevistados assinaram um Termo de Consentimento Livre e Esclarecido (TCLE).

A seleção dos hospitais foi realizada de forma intencional e buscou-se avaliar o número total dos profissionais de saúde que trabalhavam nas Unidades de Terapia Intensiva ( $\mathrm{n}=340)$. Foram incluídos no estudo médicos, enfermeiros, fisioterapeutas e técnicos de enfermagem, de ambos os sexos, que trabalhassem nas respectivas UTI's por pelo menos três meses e possuíssem vínculo empregatício com o hospital.

Os dados pessoais foram coletados a partir de uma ficha contendo quesitos sobre nome, idade, sexo, categoria profissional, carga horária de trabalho, tempo de serviço em UTI, consumo de bebida alcoólica, tabagismo, massa corporal e estatura. A massa corporal dos indivíduos foi analisada pelo uso da balança digital (G-TECH ${ }^{\oplus}$; Pernambuco, Brasil) e a estatura foi mensurada utilizando uma fita métrica (Jomarca ; São Paulo, Brasil).

Para a avaliação do NAF, utilizou-se a versão curta do Questionário Internacional de Atividade Física (IPAQ), traduzida e validada para o Brasil ${ }^{13-15}$, que classifica o indivíduo em muito ativo, ativo, irregularmente ativo ou sedentário, conforme frequência, duração e intensidade das atividades físicas semanais. Foram considerados ativos os indivíduos que atingiram os seguintes critérios: a) atividade física vigorosa com frequência igual ou superior a três dias/semana com duração igual ou maior que 20 minutos/sessão; b) atividade física moderada ou caminhada com frequência igual ou superior a cinco dias/semana e duração igual ou maior que 30 minutos/sessão; c) qualquer atividade física cuja frequência somada fosse igual ou superior a cinco dias/semana e com duração igual ou maior que 150 minutos/semana. Os sujeitos que não alcançaram os critérios supracitados foram classificados como inativos.

A qualidade de vida foi avaliada por meio do questionário Short Form Health Survey (SF-36) traduzido e validado para o Brasi $1^{16}$, que avalia oito domínios, incluindo a capacidade funcional, aspectos físicos, dor, estado geral de saúde, vitalidade, aspectos sociais, aspectos emocionais e saúde mental. As resultantes dos cálculos de cada componente variam de 0 a 100, no qual 0 é o pior e 100 o melhor resultado para cada domínio. Os questionários foram aplicados de forma individual e em um ambiente reservado.

Para testar a suposição de normalidade dos dados foi aplicado o teste de Kolmogorov-Smirnov. A comparação entre as médias foi realizada por meio da análise de variância (one-way ANOVA) com pós-teste de Tukey e teste t de Student para amostras independentes. Para avaliar as diferenças entre as proporções foram utilizados os teste de qui-quadrado e exato de Fisher, com correção de Bonferroni para comparações múltiplas. As análises foram realizadas nos pacotes estatísticos Graph Pad Prism ${ }^{\circledR}$ versão 5.0 e Winpepi versão 10.8, sendo adotado um nível de significância de 5\%.

\section{RESULTADOS}

No início do estudo, 340 profissionais preencheram os critérios de inclusão, entretanto, foram excluídos cinco profissionais que durante o período de coleta estavam 
de licença maternidade, outros treze trabalhadores que estavam de férias ou licença médica e 76 daqueles que expressaram o desejo de não participar da pesquisa. Desse modo, a amostra final foi composta por 246 profissionais de saúde dos cinco hospitais incluídos no estudo. $\mathrm{Na}$ tabela 1 estão apresentados os dados descritivos da amostra em relação à idade, índice de massa corporal e carga horária de trabalho dos profissionais de saúde.

Tabela 1 - Média e desvio padrão da idade, do IMC e da carga horária de trabalho da amostra.

\begin{tabular}{lcccc}
\hline \multicolumn{1}{c}{ Variáveis } & $\begin{array}{c}\text { Médico } \\
(n=29)\end{array}$ & $\begin{array}{c}\text { Fisioterapeuta } \\
(n=48)\end{array}$ & $\begin{array}{c}\text { Enfermeiro } \\
(n=42)\end{array}$ & $\begin{array}{c}\text { Técnico } \\
(n=127)\end{array}$ \\
\hline Idade (anos) & $35,7 \pm 8,8^{*}$ & $30,5 \pm 7,0$ & $36,4 \pm 8,9^{*}$ & $33,3 \pm 7,7^{\Uparrow}$ \\
\hline IMC $\left(\mathrm{Kg} / \mathrm{m}^{2}\right)$ & $27,2 \pm 4,1$ & $24,7 \pm 4,3$ & $24,7 \pm 4,3$ & $26,0 \pm 4,8$ \\
\hline $\begin{array}{l}\text { Carga Horária de } \\
\text { trabalho (h/sem) }\end{array}$ & $81,0 \pm 29,7^{*}$ & $62,6 \pm 36,7$ & $64,2 \pm 28,6$ & $73,8 \pm 32,6^{*}$ \\
\hline
\end{tabular}

* $p<0,05$ quando comparado aos fisioterapeutas; ๆ $p<0,05$ quando comparado aos enfermeiros. One-way ANOVA e pós-teste de Tukey.

A caracterização da amostra em relação ao gênero, NAF, tabagismo e etilismo, encontram-se na tabela 2. Quanto ao tabagismo e ao etilismo, observou-se que a maioria da amostra era não fumantes e que não apresentavam o hábito de ingerir bebida alcoólica, não existindo diferenças de proporção entre as classes profissionais.

Tabela 2 - Caracterização da amostra quanto ao gênero, prática de atividade física, tabagismo e etilismo.

\begin{tabular}{|c|c|c|c|c|c|c|}
\hline Variáveis & $\begin{array}{c}\text { Total } \\
(n=246)\end{array}$ & $\begin{array}{l}\text { Médico } \\
(n=29)\end{array}$ & $\begin{array}{l}\text { Fisioterapeuta } \\
\quad(n=48)\end{array}$ & $\begin{array}{l}\text { Enfermeiro } \\
(n=42)\end{array}$ & $\begin{array}{c}\text { Técnico } \\
(n=127)\end{array}$ & $\mathrm{p}$ \\
\hline \multicolumn{7}{|l|}{ Gênero } \\
\hline Feminino & $163(66,3)$ & $11(37,9)$ & $28(58,3)$ & $34(81,0)^{a}$ & $90(70,9)^{a}$ & \multirow{2}{*}{$0,01^{\circ}$} \\
\hline Masculino & $83(33,7)$ & $18(62,1)$ & $20(41,7)$ & $8(19,0)$ & $37(29,1)$ & \\
\hline \multicolumn{7}{|c|}{ Atividade física } \\
\hline Ativo & $93(37,8)$ & $7(24,1)^{b, c}$ & $20(41,7)$ & $9(21,4)^{b, c}$ & $57(44,9)$ & \multirow{2}{*}{$0,02^{\circ}$} \\
\hline Inativo & $153(62,2)$ & $22(75,9)$ & $28(58,3)$ & $33(78,6)$ & $70(55,1)$ & \\
\hline \multicolumn{7}{|l|}{ Tabagismo } \\
\hline Sim & $18(7,3)$ & $2(6,9)$ & $2(4,2)$ & $3(7,1)$ & $11(87)$ & \multirow{2}{*}{0,79} \\
\hline Não & $228(92,7)$ & $27(93,1)$ & $46(95,8)$ & $39(92,9)$ & $116(91,3)$ & \\
\hline \multicolumn{7}{|l|}{ Etilismo } \\
\hline Sim & $76(30,9)$ & $7(24,1)$ & $19(39,6)$ & $11(26,2)$ & $39(30,7)$ & \multirow{2}{*}{0,43} \\
\hline Não & $170(69,1)$ & $22(75,9)$ & $29(60,4)$ & $31(73,8)$ & $88(69,3)$ & \\
\hline
\end{tabular}

Técnico = técnico de enfermagem; Os valores estão expressos como números absolutos (\%). * Teste qui quadrado ou Teste exato de Fisher. a = Diferença estatística quando comparado aos médicos; $b=$ Diferença estatística quando comparado aos fisioterapeutas; $c=$ Diferença estatística quando comparado aos técnicos.

A tabela 3 apresenta os resultados obtidos dos oito domínios de QV dos profissionais avaliados, não sendo observadas diferenças significativas entre as diversas classes profissionais.

Os dados referentes à idade, IMC, carga horária de trabalho e domínios de $\mathrm{QV}$ dos profissionais de acordo com o NAF encontram-se na tabela 4. Os indivíduos considerados ativos apresentaram maiores pontuações nos domínios capacidade funcional, vitalidade e saúde mental, quando comparados aos inativos. 
Tabela 3 - Média e desvio padrão dos domínios de qualidade de vida dos profissionais de saúde.

\begin{tabular}{lccccc}
\hline \multicolumn{1}{c}{ Domínios } & $\begin{array}{c}\text { Médico } \\
(n=29)\end{array}$ & $\begin{array}{c}\text { Fisioterapeuta } \\
(n=48)\end{array}$ & $\begin{array}{c}\text { Enfermeiro } \\
(n=42)\end{array}$ & $\begin{array}{c}\text { Técnico } \\
(n=127)\end{array}$ & Valor-p* \\
\hline CF & $86,0 \pm 12,6$ & $87,8 \pm 13,8$ & $79,5 \pm 22,0$ & $79,1 \pm 20,9$ & 0,08 \\
\hline LAF & $86,2 \pm 28,0$ & $85,5 \pm 24,9$ & $78,0 \pm 29,3$ & $80,1 \pm 28,7$ & 0,26 \\
\hline DOR & $76,9 \pm 19,3$ & $73,8 \pm 19,2$ & $69,6 \pm 21,2$ & $67,0 \pm 22,7$ & 0,07 \\
\hline EGS & $77,3 \pm 19,1$ & $77,8 \pm 19,6$ & $75,2 \pm 19,7$ & $74,4 \pm 18,2$ & 0,55 \\
\hline VITALIDADE & $68,3 \pm 18,9$ & $63,8 \pm 21,1$ & $65,4 \pm 25,2$ & $67,3 \pm 23,4$ & 0,78 \\
\hline AS & $83,2 \pm 21,0$ & $83,3 \pm 18,7$ & $80,4 \pm 16,8$ & $80,3 \pm 21,7$ & 0,58 \\
\hline AE & $95,7 \pm 14,2$ & $80,5 \pm 34,9$ & $80,6 \pm 30,4$ & $83,7 \pm 30,8$ & 0,09 \\
\hline SM & $76,5 \pm 11,4$ & $74,7 \pm 14,4$ & $76,3 \pm 12,3$ & $76,8 \pm 15,2$ & 0,77 \\
\hline
\end{tabular}

$\mathrm{CF}=$ capacidade funcional; $\mathrm{LAF}=$ limitação por aspectos físicos; $\mathrm{EGS}=$ estado geral de saúde; $\mathrm{AS}=$ aspecto social; $\mathrm{AE}=$ aspecto emocional; $\mathrm{SM}=$ saúde mental; * one-way ANOVA .

Tabela 4 - Idade, IMC, carga horária de trabalho e domínios de qualidade de vida de acordo com o nível de atividade física dos profissionais avaliados.

\begin{tabular}{|c|c|c|c|}
\hline \multirow[b]{2}{*}{ Variáveis } & \multicolumn{2}{|c|}{ Nível de Atividade física } & \multirow[b]{2}{*}{ Valor-p* } \\
\hline & $\begin{array}{c}\text { Ativo } \\
(n=93)\end{array}$ & $\begin{array}{c}\text { Inativo } \\
(n=153)\end{array}$ & \\
\hline Idade (anos) & $33,7 \pm 8,4$ & $33,5 \pm 8,0$ & 0,89 \\
\hline IMC $\left(\mathrm{Kg} / \mathrm{m}^{2}\right)$ & $25,7 \pm 4,1$ & $25,7 \pm 5,0$ & 0,97 \\
\hline Carga horária de trabalho (h/sem) & $67,7 \pm 31,1$ & $72,1 \pm 34,1$ & 0,38 \\
\hline Capacidade funcional & $86,1 \pm 17,4$ & $79,0 \pm 20,0$ & 0,01 \\
\hline Limitação por aspectos físicos & $86,3 \pm 22,7$ & $78,6 \pm 30,5$ & 0,12 \\
\hline Dor & $71,5 \pm 21,4$ & $69,0 \pm 21,8$ & 0,47 \\
\hline Estado geral de saúde & $77,1 \pm 16,3$ & $74,6 \pm 20,1$ & 0,58 \\
\hline Vitalidade & $71,2 \pm 18,3$ & $62,9 \pm 25,0$ & 0,01 \\
\hline Aspecto social & $84,9 \pm 17,7$ & $79,0 \pm 21,3$ & 0,05 \\
\hline Aspecto emocional & $89,5 \pm 22,9$ & $80,6 \pm 33,7$ & 0,13 \\
\hline Saúde mental & $80,1 \pm 13,3$ & $74,0 \pm 14,2$ & 0,01 \\
\hline
\end{tabular}

As variáveis estão expressas como médias \pm desvios padrão. * Teste $t$ de Student para amostras independentes.

\section{DISCUSSÃO}

O presente estudo teve como objetivo verificar se os domínios da qualidade de vida de profissionais de saúde que atuam em Unidades de Terapia Intensiva diferem em função do nível de atividade física. Os resultados desta pesquisa revelaram que os profissionais classificados como ativos apresentaram maiores escores de QV, nos domínios capacidade funcional, vitalidade e saúde mental.

Os resultados sobre o NAF dos profissionais avaliados revelaram que os médicos e enfermeiros foram os profissionais que apresentaram menor proporção de sujeitos ativos, apresentando inclusive valores inferiores a média nacional de $36 \%{ }^{17}$. Jatkinson et al., ${ }^{18}$ verificaram que os médicos, mesmo realizando caminhadas durante sua jornada de trabalho, não conseguiam suprir uma proporção substancial das necessidades diárias, tornando-se necessária a prática complementar de atividade física.

Por outro lado, os técnicos de enfermagem e os fisioterapeutas apresentaram valores superiores aos médicos, enfermeiros e inclusive em relação à proporção nacional. Uma possível explicação para a maior proporção de indivíduos ativos 
entre esses profissionais, pode estar relacionada à influência das atividades desenvolvidas em suas rotinas de trabalho nos escores do IPAQ.

Nas suas rotinas de trabalho, os técnicos são responsáveis pelo deslocamento de pacientes, cuidados com higiene pessoal, administração de fármacos e transporte de diferentes equipamentos. Todas essas atividades demandam maior gasto energético, pois exigem que esses profissionais percorram maiores distâncias dentro do ambiente hospitalar, seja caminhando, subindo e descendo escadas, bem como realizando atividades vigorosas que exigem força física, como a transferência de pacientes e tarefa de banho no leito. Explicação semelhante pode ser pensada para os fisioterapeutas que utilizam a mobilização dos pacientes em suas rotinas como a transferência para cadeira, poltrona, ortostatismo, além da deambulação.

O sedentarismo vem sendo visto como um problema de saúde pública no mundo, particularmente no Brasil ${ }^{19,20}$. Nesse sentido, cabe salientar que a proporção de indivíduos ativos verificados no presente estudo ainda está muito longe do ideal e estratégias devem ser implementadas para alterar esses achados. A AF desempenha papel importante para o desenvolvimento motor dos indivíduos, melhora as relações interpessoais nos ambientes familiares e de trabalho, além de melhorar a $\mathrm{QV}^{10}$.

De acordo com Matsudo et al., ${ }^{21}$ o sedentarismo não representa apenas alto risco para a saúde do indivíduo, mas elevados custos econômicos para os mesmos, para a família e sociedade. Em um ambiente de terapia intensiva os profissionais precisam lidar com a vida e a morte dos seus pacientes o tempo inteiro, e muitas vezes eles não foram preparados psicologicamente e emocionalmente para lidar com estas situações. Por isto, esta população merece atenção especial, no sentido da prevenção da sua saúde.

Além disso, cabe ressaltar o quanto pode ser penoso, insalubre e estressante o ambiente hospitalar, e quanto pode ser desgastante lidar com o sofrimento alheio e cuidar de enfermos. A elevada carga horária de trabalho, a baixa remuneração e o pluriempregos podem levar ao adoecimento do profissional, aumento de riscos de acidentes trabalho e diminuição da $\mathrm{QV}^{22}$. Dessa forma, o incentivo à prática de $\mathrm{AF}$ por parte do hospital, com programas que estimulem a prática esportiva pode ser um ponto fundamental na promoção da saúde e manutenção da QV dos seus colaboradores.

Oler et al., ${ }^{4}$ estudaram enfermeiros do centro cirúrgico de um Hospital escola, e relataram que a $\mathrm{QV}$ destes profissionais estava comprometida e que o domínio mais afetado foi a dor, seguido pela vitalidade, aspecto social e com igual frequência pelo aspecto físico e saúde mental. Para os referidos autores ${ }^{4}$ isso pode ser justificado pelo desgaste físico e mental que essa população é submetida diariamente na sua rotina de trabalho. Corroborando esses achados, outros estudos ${ }^{5-7}$ também observaram diminuição da QV em médicos e enfermeiros que atuam em diferentes ambientes hospitalares. No entanto, nenhum desses estudos buscou avaliar a associação entre QV e NAF, o que dificulta a comparação e discussão dos dados do presente estudo.

Segundo o DIEESE (Departamento Intersindical de Estatística e Estudos Socioeconômicos ${ }^{23}$, no Brasil a carga horária semanal de um trabalhador formal é de 44 horas, observando os desse estudo, nota-se que a média da jornada de trabalho dessa população, chega a ser quase o dobro do que ocorre na população geral. Esse fator juntamente com os níveis de inatividade física, podem ter contribuído para a diferença dos domínios referentes à $\mathrm{QV}$, entre os ativos e inativos, e revela o quanto estes profissionais estão sujeitos a altas horas de trabalho. Um documento, fornecido pelo DIEESE ${ }^{23}$, retrata o debate do órgão sobre a redução da jornada de trabalho semanal no congresso nacional de 44 horas para 40 horas, o qual aponta vários fatores explicando que a redução da jornada de trabalho possibilitaria aos 
trabalhadores dedicar mais tempo para o convívio familiar, o estudo, o lazer e o descanso, melhorando a $\mathrm{QV}$ dos profissionais.

Para Tamayo ${ }^{24}$, o nível de estresse em profissionais que não incluem na sua rotina $\mathrm{AF}$ regular, é maior do que seus pares que praticam. No tocante a prática de $\mathrm{AF}$, pessoas com este hábito demonstram maior energia e disposição para a realização de suas atividades da rotina de trabalho ${ }^{8}$. Nesta pesquisa, os domínios capacidade vital, vitalidade e saúde mental encontraram-se maiores nos indivíduos ativos, indicando melhores escores de QV. Embora inúmeros estudos tenham avaliado a QV e/ou o NAF em trabalhadores de diferentes setores da economia ${ }^{25-28}$, ainda são escassos os estudos que buscam avaliar a associação dessas variáveis em profissionais de saúde que trabalham em ambiente hospitalar, especificamente em UTI's, o que limita a discussão e comparação desses resultados.

Esses achados sugerem que a prática regular da $\mathrm{AF}$ por ser um grande aliado na busca da melhora ou manutenção de vários indicadores de saúde e $\mathrm{QV}^{29,30}$. Resultados positivos têm sido observados na literatura, demonstrando que a inserção de práticas de $\mathrm{AF}$ no ambiente laboral tem colaborado de forma significativa para melhora nos domínios da saúde e na percepção de $\mathrm{QV}^{25}$. Diante disso, medidas simples e baratas como a avaliação do nível de AF e o acompanhamento destes profissionais podem prevenir problemas futuros de saúde, diminuindo o absenteísmo hospitalar, aumentando o rendimento pessoal e consequentemente possibilitando a melhora da qualidade do serviço prestado à população.

Contudo, é importante destacar algumas limitações em relação ao presente estudo, principalmente no que diz respeito ao número e seleção amostral. Por ter avaliado uma amostra intencional de apenas cinco hospitais da RMR, os dados deste estudo não podem ser generalizados para outras localidades. Além disso, outros fatores, tais como trabalho, segurança, moradia, serviços de saúde, dinheiro, qualidade do ar e serviços de transporte que podem influenciar a percepção de QV não foram avaliados. Desse modo, sugere-se a realização de estudos futuros que busquem avaliar a associação desses diferentes fatores com a $\mathrm{QV}$ e $\mathrm{AF}$, em amostras maiores e em diferentes localidades. Porém, cabe destacar a importância do presente do estudo, uma vez que os resultados aqui descritos podem subsidiar o desenvolvimento de pesquisas futuras que procurem comparar diferentes intervenções de promoção à saúde do trabalhador e seu impacto nos domínios da qualidade de vida em profissionais de saúde que atuam em ambiente hospitalar.

Em suma, pode-se concluir com os resultados deste estudo que os escores de QV, principalmente nos domínios capacidade funcional, vitalidade e saúde mental diferiram entre os profissionais de saúde classificados como ativos e inativos, sendo verificado melhores níveis de $\mathrm{QV}$ nos indivíduos fisicamente ativos. Assim, constata-se a importância de ser dada maior atenção por parte dos gestores e dos setores de medicina do trabalho para a inclusão de programas de incentivo à prática de atividade de física para esses profissionais, no intuito de tentar minimizar ou evitar os elevados níveis de inatividade física e suas consequentes morbidades.

\section{Agradecimentos / Financiamento}

Agradecemos a todos os profissionais e coordenadores dos Hospitais que gentilmente participaram e contribuíram com a pesquisa.

\section{Contribuição dos autores}

Afonso Celso de Farias Acioli Neto e Luciana Carvalho de Menezes participaram de todas as etapas de condução do estudo e redação do artigo, Eduardo Eriko Te- 
nório de França, Emilia Chagas Costa e Ana Carolina Rodarti Pitangui contribuíram revisando criticamente o manuscrito e realizando as análises estatísticas, Flávio Maciel Dias de Andrade e Rodrigo Cappato de Araújo participaram da revisão da literatura e revisão do manuscrito além de ter contribuído na análise estatística e Marco Aurélio de Valois Correia Junior como coordenador do estudo e orientador.

\section{REFERÊNCIAS}

1. The WHOQOL Group. The World Health Organization quality of life assessment (WHOQOL): position paper from the World Health Organization. Soc Sci Med. 1995;41(10):1403-1409.

2. Minayo MCS, Hartz ZMA, Buss PM. Qualidade de vida e saúde: um debate necessário. Ciênc. Saúde Colet . 2000;5(1):7-18.

3. Alleyne GAO. Health and the quality of life. Rev Panam Salud Publica 2001;9(1):1-6.

4. Oler FG, Jesus AF, Barboza DB, Domingos NAM. Qualidade de vida da equipe de enfermagem do Centro Cirúrgico. Arq Ciênc Saúde. 2005;12(2):102-110.

5. Siqueira Júnior AC, Siqueira FPC, Gonçalves BGOG. O trabalho noturno e a qualidade de vida dos profissionais de enfermagem. REME Rev Min Enferm. 2006;10(1):41-45.

6. Paschoa S, Zanei SSV, Whitaker IY. Qualidade de vida dos trabalhadores de enfermagem de Unidades de Terapia Intensiva. Acta Paul Enferm. 2007;20(3):305-310.

7. Fogaça MC, Carvalho WB, Nogueira-Martins LA. Estudo preliminar sobre a qualidade de vida de médicos e enfermeiros intensivistas pediátricos e neonatais. Rev. esc. enferm. USP. 2010;44(3):708-712.

8. Macedo CSG, Garavello JJ, Oku EC, Miyagusuku, FH, Agnoll PD, Nocetti PM. Benefícios do exercício físico para a qualidade de vida. Rev Bras Ativ Fís Saúde. 2003;8(2):19-27.

9. Toscano JJO, Oliveira ACC. Qualidade de vida em idosos com distintos níveis de atividade física. Rev Bras Med Esporte. 2009;15(3):169-173

10. Negrão CE, Tinucci T, Rondon MUPB. Estratégias para mudanças de hábitos de vidaExercício físico. Cardio Sintética 1999;12:13-15.

11. Ferrareze MVG, Ferreira V, Carvalho AMP. Percepção do estresse entre enfermeiros que atuam em Terapia Intensiva. Acta Paul Enferm, 2006;19(3):310-315.

12. Shimizu HE, Ciampone MHT. Sofrimento e prazer no trabalho vivenciado pelas enfermeiras que trabalham em Unidades de Terapia Intensiva em um hospital escola. Rev Esc Enfermagem USP, 1999;33(1):95-106.

13. Guedes DP, Lopes CC, Guedes JERP. Reprodutibilidade e validade do questionário Internacional de atividade física em adolescentes. Rev Bras Med. Esporte. 2005;11(2):151-158.

14. Craig CL, Marshall AL, Sjöström M, Bauman AE, Booth ML, Ainsworth BE, et al. International Physical Activity Questionnaire: 12-Country Reliability and Validity. Med Sci Sports Exerc. 2003;35(8):1381-95.

15. Matsudo S,Araújo T,Matsudo V, Andrade D, Andrade E, Oliveira C,Braggion G. Questionário Internacional de Atividade Física (IPAQ): estudo de validade e reprodutibilidade no Brasil. Rev Bras Ativ Fís Saúde. 2001;6(2):5-12.

16. Ciconelli RM, Ferraz MB, Santos W, Meinão I, Quaresma MR. Tradução para a língua portuguesa e validação do questionário genérico de avaliação de qualidade de vida SF-36 (Brasil SF-36). Rev.Bras.Reumatol. 1999;39(3):141-150.

17. Pesquisa nacional por amostra de domicílios 2008. Brasil. Rio de Janeiro: IBGE, v. 29, 2009. [Acesso mar 2013]. Disponível em: http://www.ibge.gov.br/home/estatistica/populacao/ panorama_saude_brasil_2003_2008/PNAD_2008_saude.pdf.

18. Jatkinson J, Goody RB, Walter CA. Walking at work: a pedometer study assessing the activity levels of doctors. Scott Med J. 2005;50(2):73-74.

19. Carvalho MC, Ricarte IF, Rocha CHL, Maia RB, Silva VB, Veras AB. Pressão arterial, excesso de peso e nível de atividade física em estudantes de universidade pública. Arq Bras Cardiol, 2010; 95(2):192-199.

20. Hallal PC, Dumith SM, Bastos JP, Reichert FF, Siqueira FV, Azevedo MR. Evolução da pesquisa epidemiológica em atividade física no Brasil: revisão sistemática. Rev Saúde Pública, 2007;41(3):453-460. 
21. Matsudo SM, Matsudo VR, Araújo T, Andrade D, Andrade E, Oliveira L. Nível de atividade física da população do Estado de São Paulo: Análise de acordo com o gênero, idade, nível socioeconômico, distribuição geográfica e de conhecimento. Rev Bras Ciên Mov. 2002; 10(4):41-50.

22. Lima Júnior JHV, Ésther AB. Transições, prazer e dor no trabalho de enfermagem. Rev. Adm. Emp. 2001; 41(3):20-30.

23. Departamento intersindical de estatística e estudos socioeconômicos (DIEESE). São Paulo. Brasil. 2010. [Acesso out 2013]. Disponível em: http://www.dieese.org.br/notatecnica/2009/ notatec85ArgumentosReduzirJornada.pdf

24. Tamayo A. Prioridades axiológicas, atividade física e estresse ocupacional. Rev. Adm. Contemp.2001; 5(3):127-147

25. Grande AJ, Silva V, Manzatto LRTBX, Martins GC, Vilela Junior GB. Comparação de intervenções de promoção à saúde do trabalhador: ensaio clínico controlado randomizado por cluster. Rev. bras. cineantropom. desempenho hum. 2013;15(1):27-37.

26. Siqueira FCV, Nahas MV, Facchini LA, Piccini RX, Tomasi E, Thumé E, et al. Atividade física em profissionais de saúde do Sul e Nordeste do Brasil. Cad. Saúde Pública. 2009;25(9):19171928.

27. Farah BQ, Barros MVG, Farias Júnior JC, Ritti-Dias RM, Lima RA, Barbosa JPAS et al. Percepção de estresse: associação com a prática de atividades físicas no lazer e comportamentos sedentários em trabalhadores da indústria. Rev. bras. educ. fís. esporte. 2013; 27(2):225-234.

28. Brown DW, Brown DR, Heath GW, Balluz L, Giles WH, Ford ES et al. Associations between physical activity dose and health-related quality of life. Med sci. Sports Exerc 2004;36(5):890-896.

29. Siqueira FCV, Nahas MV, Facchini LA, Piccini RX, Tomasi E, Thumé E, et al. Atividade física em profissionais de saúde do Sul e Nordeste do Brasil. Cad. Saúde Pública. 2009;25(9):1917-1928.

30. Silva RS, Silva I, Silva RA, Souza L, Tomasi E. Atividade física e qualidade de vida. Rev. Ciên Saúde Colet. 2010;15(1):115-120.

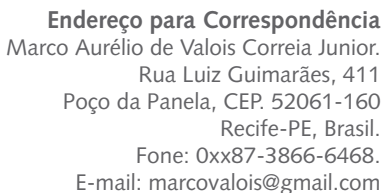

